Beat Knechtle ist der dritte neue Professor 2015 am IHAMZ

\author{
Rosemann, T
}

Posted at the Zurich Open Repository and Archive, University of Zurich ZORA URL: https://doi.org/10.5167/uzh-116944

Journal Article

Published Version

Originally published at:

Rosemann, T (2015). Beat Knechtle ist der dritte neue Professor 2015 am IHAMZ. PrimaryCare:391. 


\section{Beat Knechtle ist der dritte neue Professor 2015 am IHAMZ}

Thomas Rosemann

An den Universitäten gibt es im deutschsprachigen Raum zwei Wege zum Professorentitel. Der klassische ist die Berufung auf eine institutionalisierte Professur, die in der Planung der Universität als Ordinariat oder Extraordinariat fest verankert ist und mittels internationaler Ausschreibung kompetitiv besetzt wird. Der zweite Weg ist die sogenannte "Titularpropfessur", die quasi in Anerkennung der wissenschaftlichen Leistungen nach einem bestimmten Zeitraum - in Zürich 6 Jahre - von habilitierten Kollegen beantragt werden kann, wenn sie eine fortwährende Forschungs- und Lehrtätigkeit nachweisen können. Titularprofessoren

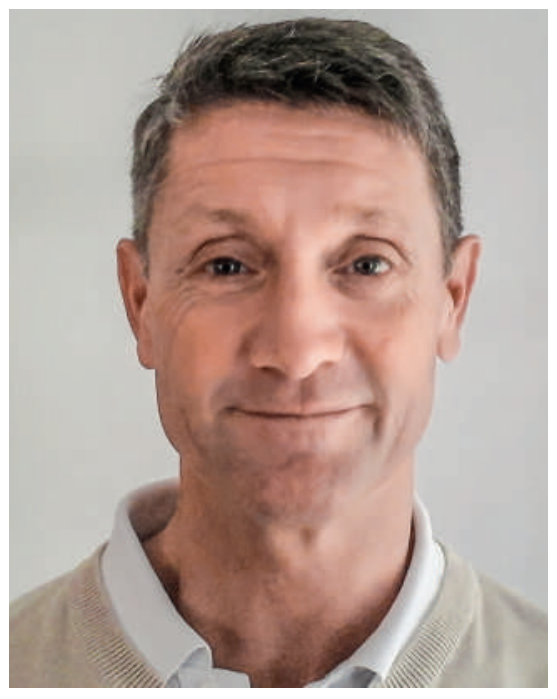

Beat Knechtle haben jedoch kein Promotionsrecht und sind auch nicht Fakultätsmitglieder.

Am Institut für Hausarztmedizin wurde im Frühjahr dieses Jahres mit der Berufung von PD Dr. Oliver Senn eine weitere institutionalisierte Professur besetzt, kurz darauf wurde eine Titularprofessur an PD Dr. Claudia Steurer-Stey verliehen. Aller guten Dinge sind aber drei; daher freut es uns, dass wir nun mit PD Dr. Beat Knechtle schon den dritten neuen Professor in diesem Jahr am Institut begrüssen dürfen.

Beat Knechtle wurde am 26. Mai 1964 in Appenzell (AI) geboren, das Medizinstudium absolvierte er an den Universitäten von Fribourg, Basel und Zürich, wo er 1991 mit dem Staatsexamen abschloss. Seit 2004 arbeitet Beat Knechtle als Grundversorger im Gesundheitszentrum St. Gallen. Seit Gründung des IHAMZ 2008 forscht er intensiv aus der Praxis heraus mit uns und engagiert sich als Lehrarzt für das Institut. Allein seit seiner Habilitation 2009 veröffentlichte er 204 (!) wissenschaftliche Originalarbeiten. Er ist leidenschaftlicher Extremausdauersportler, und so wundert es auch nicht, dass er sich in vielen Forschungsarbeiten zum Beispiel damit auseinandersetzt, wie man am meisten Fett verbrennt oder am besten fit wird. Aspekte, die auch in der hausärztlichen Beratung einen Stellenwert haben.

Wir gratulieren Beat ganz herzlich zur Anerkennung seiner wissenschaftlichen Ausdauerleistung durch die Verleihung der Titularprofessur. Insbesondere freut mich, dass Beat den Beweis angetreten hat, dass es auch aus der hausärztlichen Praxis heraus möglich ist, sich zu habilitieren und Professor zu werden. Gerne haben ich und das IHAMZ ihn dabei unterstützt! 